\title{
Transmembrane and coiled-coil domain family 3 (TMCC3) regulates breast cancer stem cell and AKT activation
}

\author{
Ya-Hui Wang ${ }^{1}$ Y Yu-Tzu Chan ${ }^{1} \cdot$ Tsai-Hsien Hung ${ }^{1}$ - Jung-Tung Hung ${ }^{1} \cdot$ Ming-Wei Kuo ${ }^{1}$ Sheng-Hung Wang ${ }^{1}$. \\ Yenlin Huang $^{2,3} \cdot$ Yu-Ju Lin ${ }^{1} \cdot$ Shin-Cheh Chen $\mathbb{1}^{4} \cdot$ Jyh-Cherng Yu ${ }^{5} \cdot$ Jen-Chine Wu ${ }^{1} \cdot$ John Yu $\mathbb{D}^{1,3} \cdot$ Alice L. Yu $\mathbb{D}^{1,3,6}$
}

Received: 3 September 2020 / Revised: 17 February 2021 / Accepted: 19 February 2021 / Published online: 19 March 2021

(c) The Author(s) 2021. This article is published with open access

\begin{abstract}
Cancer stem cells (CSC) play a pivotal role in cancer metastasis and resistance to therapy. Previously, we compared the phosphoproteomes of breast cancer stem cells (BCSCs) enriched subpopulation and non-BCSCs sorted from breast cancer patient-derived xenograft (PDX), and identified a function unknown protein, transmembrane and coiled-coil domain family 3 (TMCC3) to be a potential enrichment marker for BCSCs. We demonstrated greater expression of TMCC3 in BCSCs than non-BCSCs and higher expression of TMCC 3 in metastatic lymph nodes and lungs than in primary tumor of breast cancer PDXs. TMCC 3 silencing suppressed mammosphere formation, ALDH activity and cell migration in vitro, along with reduced tumorigenicity and metastasis in vivo. Mechanistically, we found that AKT activation was reduced by TMCC 3 silencing, but enhanced by TMCC 3 overexpression. We further demonstrated that TMCC 3 interacted directly with AKT through its 1-153 a.a. domain by cell-free biochemical assay in vitro and co-immunoprecipitation and interaction domain mapping assays in vivo. Based on domain truncation studies, we showed that the AKT-interacting domain of TMCC3 was essential for TMCC3-induced AKT activation, self-renewal, and metastasis. Clinically, TMCC3 mRNA expression in 202 breast cancer specimens as determined by qRT-PCR assay showed that higher TMCC 3 expression correlated with poorer clinical outcome of breast cancer, including early-stage breast cancer. Multivariable analysis identified $T M C C 3$ expression as an independent risk factor for survival. These findings suggest that TMCC 3 is crucial for maintenance of BCSCs features through AKT regulation, and TMCC3 expression has independent prognostic significance in breast cancer. Thus, TMCC3 may serve as a new target for therapy directed against CSCs.
\end{abstract}

\section{Introduction}

Breast cancer is the most common cancer among women worldwide. The majority of breast cancer deaths occur as a result of recurrent or metastatic disease rather than from the

Supplementary information The online version contains supplementary material available at https://doi.org/10.1038/s41388021-01729-1.

John Yu

johnyu@gate.sinica.edu.tw

$\triangle$ Alice L. Yu

a1yu@ucsd.edu

1 Institute of Stem Cell and Translational Cancer Research, Chang Gung Memorial Hospital at Linkou, Taoyuan, Taiwan

2 Department of Anatomic Pathology, Chang Gung Memorial Hospital at Linkou, Taoyuan, Taiwan effects of the primary tumor [1]. The existences of cancer stem cells (CSCs) have been demonstrated in a variety of human cancers, including breast cancer [2, 3]. In 2003, AlHajj et al. reported that breast cancer stem cells (BCSCs) were enriched in the $\mathrm{CD} 24^{-} \mathrm{CD} 44^{+}$subpopulation of breast cancer [2]. Subsequently, Ginestier et al. demonstrated that tumor cells with high aldehyde dehydrogenase activity (ALDH) which mediated the conversion of retinaldehyde to retinoic acid, harbored stem/progenitor properties $[1,4,5]$.

3 Chang Gung University, Taoyuan, Taiwan

4 General Surgery Department, Chang Gung Memorial Hospital at Linkou, Taoyuan, Taiwan

5 Division of General Surgery, Department of Surgery, Tri-Service General Hospital, National Defense Medical Center,

Taipei, Taiwan

6 Department of Pediatrics, University of California in San Diego, San Diego, CA, USA 
These CSCs possessed the capacity for self-renewal, differentiation, and displayed resistance to chemotherapeutic agents and radiation, which might contribute to tumor relapse years after the clinical remission [6]. Thus, it will be important to understand the molecular characteristics of CSCs that may lead to the development of novel targets for CSC-directed therapy.

Mounting data have identified many dysregulated signaling pathways in BCSCs, including the JAK/STAT, Hedgehog, Wnt, Notch, PI3K/PTEN, and NF-kB [7-13]. In our previous study, we also demonstrated that IGF-1R/PI3K/AKT/mTOR pathway was crucial for BCSC maintenance [14]. In the pursuit of novel molecular signature of BCSCs, we performed comparative phosphoproteomic analysis of BCSCs and nonBCSCs [15]. Our interest in the novel protein, transmembrane and coiled-coil domain family 3 (TMCC3) was piqued by phosphoproteomic data showing greater phosphorylation of TMCC 3 in BCSCs than in non-BCSCs.

Genes encoding putative proteins of the transmembrane and coiled-coil domain (TMCC) family have been found in many organisms. The TMCC family consists of three putative proteins (TMCC1-3) that are conserved from nematode to human [16]. However, the properties and functions of TMCC proteins are little known. It was reported that TMCC1 localizes to the rough ER and is crucial for ER-associated bud fission [16, 17]. TMCC2 is a neuronal, ER-located protein, and the interaction between TMCC 2 and apoE contributes to amyloid- $\beta$ protein precursor metabolism in Alzheimer's disease [18]. Impaired function of dementin (Drosophila orthologue of TMCC2) causes neurodegeneration and early death in Drosophila [19]. TMCC3 is first cloned from human normal brain tissue. It is also predicted as an integral membrane protein and localized in ER [17, 20]. However, the function of TMCC3 remains elusive. In this study, we provide first evidence for the important roles of TMCC 3 in self-renewal, metastasis, and tumorigenicity of BCSCs and AKT activation.

\section{Results}

\section{BCSCs express higher levels of TMCC3 protein than non-BCSCs}

Previously, we established several patient-derived xenograft tumors (PDXs) of breast cancer and identified their enrichment markers for BCSCs by determining the frequency of tumor-initiating cells, after sorting by fluorescence-activated cell sorter. Mice were injected with serial dilution of sorted cells to identify the markers for enrichment of BCSC. The supporting evidence for $\mathrm{CD} 24^{-} \mathrm{CD} 44^{+}$as $\mathrm{BCSC}$ enrichment marker for BC0145 was published previously [14]. In our previous phosphoproteomic analysis of BCSCs and
non-BCSCs sorted from BC0145 PDX, the functions of $21 \%$ of 455 phosphoproteins upregulated in BCSCs, including TMCC3, were unknown (Fig. 1a) [15]. In replicate phosphoproteomic studies, greater phosphorylated TMCC3 at Serine 216 was noted in BCSCs than in non-BCSCs by 1.5 and 3.8 folds (Supplementary Fig. S1a). TMCC3 belongs to the TMCC family that includes TMCC1 -3 and contains two coiled-coil domains of the $\mathrm{N}$-terminal region and two transmembrane domains of the C-terminal region (Supplementary Fig. S1b). Sequence alignment of TMCC3 from various vertebrate species shows several highly conserved regions (Supplementary Fig. S1c), suggesting the important roles of this protein in the vertebrates.

To confirm the differential expression levels of TMCC 3 protein in BCSCs enriched populations and non-BCSCs, we determined BCSC enrichment markers in three breast cancer PDXs. Previously, $\mathrm{H} 2 \mathrm{k}^{\mathrm{d}-} \mathrm{CD} 24^{-} \mathrm{CD} 44^{+}$cell population was shown to be enriched in BCSC of BC0145 PDX [14]. For BC0350R1 and BC0634 PDXs, aldehyde dehydrogenase $\left(\mathrm{H}_{2} \mathrm{k}^{\mathrm{d}-} \mathrm{ALDH}^{\mathrm{h}}\right)$ activity was characterized as the BCSC enrichment marker base on tumorigenicity assay in vivo (Supplementary Table S1). Using the aboveidentified BCSC enrichment markers, BCSCs and nonBCSCs were sorted from these three PDXs for western blotting. As shown in Fig. 1b, the protein levels of TMCC3 were higher in BCSCs than non-BCSCs by $10.7,9.8$, and 8.9 folds, for BC0145, BC0350R1, and BC0634 tumors, respectively.

To facilitate in vitro investigation of the involvement of TMCC 3 in BCSCs, AS-B145 and AS-B634 cells were cultured from sorted $\mathrm{H} 2 \mathrm{~K}^{\mathrm{d}-} \mathrm{CD} 24^{-} \mathrm{CD} 44^{+}$cells of BC0145 and $\mathrm{H} 2 \mathrm{~K}^{\mathrm{d}-} \mathrm{ALDH}^{\mathrm{h}}$ cells of BC0634 PDXs, respectively, for limited passages ( $\leq 10$ passages) $[14,15]$. Using these two short-term cultured cells and established breast cancer cell lines, we compared the expression levels of TMCC3 in mammosphere-cultured (Sphere) and monolayer-cultured (2D) cells. As shown in Fig. 1c, TMCC3 protein expression was greater in mammosphere-cultured cells by 1.5, 3.8, 6.0, and 3.3 folds in MCF7, MDA-MB231 (MB231), AS-B145, and AS-B634, respectively. These findings suggest that TMCC 3 may play a role in the maintenance of BCSCs.

\section{TMCC3 contributes to mammosphere formation and ALDH activity in BCSCs}

To delineate the roles of TMCC3 in BCSCs, we examined the effects of changing TMCC3 expression on the mammosphere formation and ALDH activity which are important features of BCSCs [1, 2, 21-23] by lentivirus-mediated silencing or overexpression. Upon silencing of TMCC 3 by shRNA \#A, \#B, or \#C clones, TMCC3 mRNAs were reduced to $0.24 \pm 0.02,0.09 \pm 0.02$, and $0.3 \pm 0.01$ folds, respectively, of control shRNA transfected AS-B145 cells; 
a
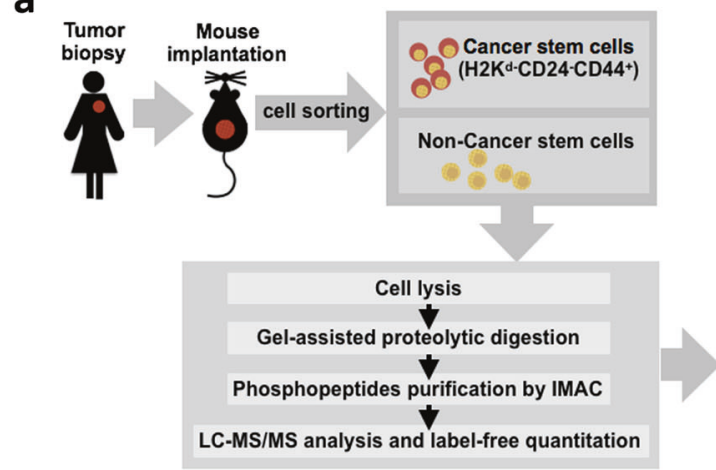

b

BC0145 BC0350R1 BC0634

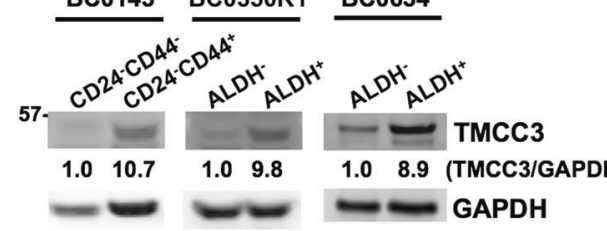

d

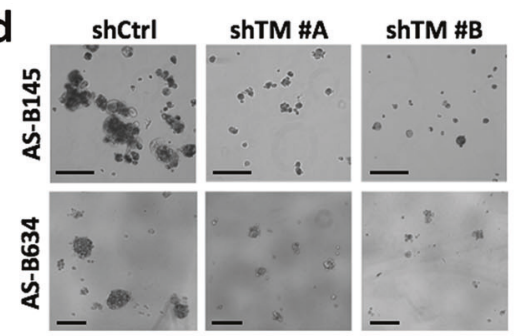

f AS-B145
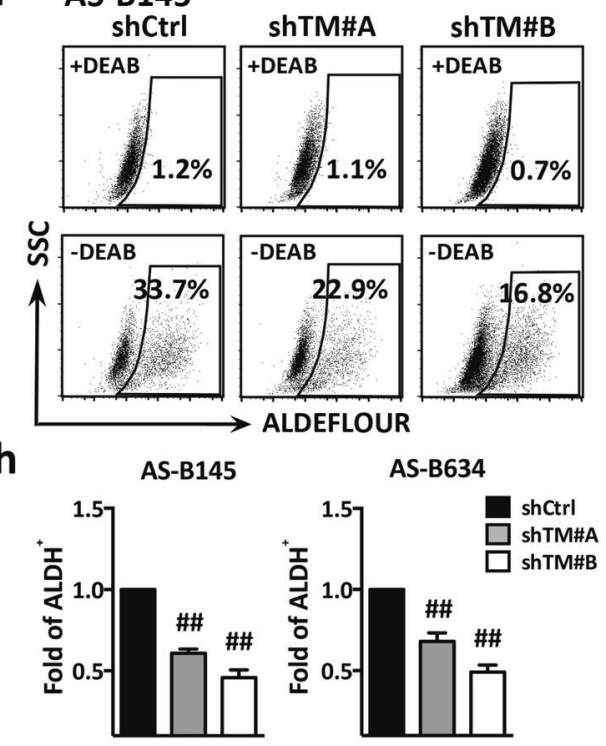

and to $0.42 \pm 0.02,0.23 \pm 0.06$, and $0.82 \pm 0.12$ folds, respectively, in AS-B634 (Supplementary Fig. S2a). Western blotting confirmed the reduction of TMCC3 protein to negligible levels (Supplementary Fig. S2b). shRNATMCC 3 \#A and \#B clones, which provided better knockdown efficiency were chosen for further studies. Stable clone of TMCC3 overexpression was established using

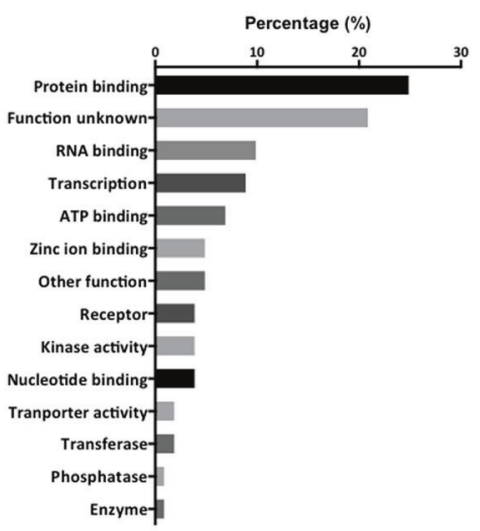

C

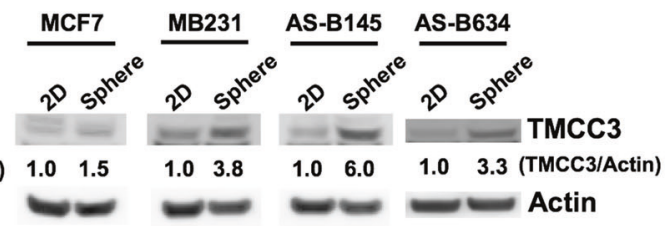

e
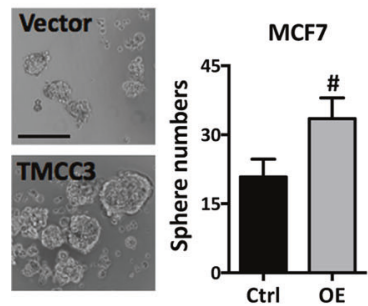

g
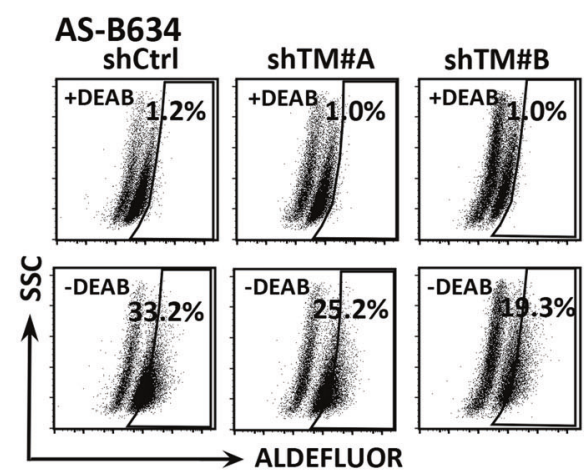

i
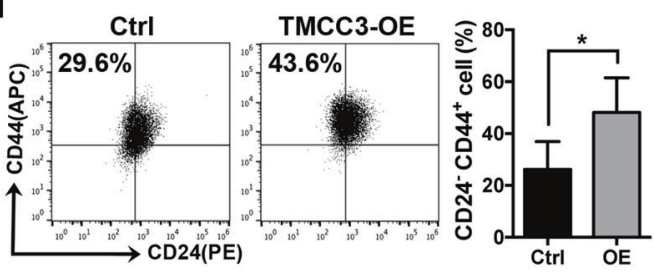

MCF7, which expressed low level of TMCC3 (Supplementary Fig. S2c, d). As shown in Fig. 1d, mammosphere numbers were significantly lower in shRNA-TMCC3 \#A and \#B transduced AS-B145 $(1.0 \pm 0.4$ and $0.2 \pm 0.2)$ and AS-B634 $(0.2 \pm 0.2$ and $0.2 \pm 0.2)$ than in shRNA-control (7.4 \pm 1.5 and $18.5 \pm 0.8, n=6, p<0.0001)$. To confirm the participation of TMCC3 in mammosphere formation, we 
Fig. 1 TMCC3 is highly expressed in BCSCs and crucial for BCSC maintenance. a Workflow illustration of phosphoproteomic analysis using BCSCs and non-BCSCs of BC0145 PDX tumor to discover function unknown phosphoproteins in BCSCs. $\mathbf{b}$ The protein levels of TMCC 3 in BCSCs and non-BCSCs FACS-sorted from breast cancer PDX tumors, BC0145, BC0350R1, and BC0634 using H2k ${ }^{\mathrm{d}-} \mathrm{CD} 24$ ${ }^{-} \mathrm{CD} 44^{+}(\mathrm{BC} 0145)$ and $\mathrm{H} 2 \mathrm{k}^{\mathrm{d}-} \mathrm{ALDH}^{+}(\mathrm{BC} 0350 \mathrm{R} 1$ and $\mathrm{BC} 0634)$, respectively. $\mathbf{c}$ The protein levels of TMCC3 in MCF7, MDA-MB231 (MB231), AS-B145, and AS-B634 cultured as monolayer (2D) and mammosphere culture (Sphere) condition, as determined by western blotting. d The representative images of mammosphere in TMCC3 silenced AS-B145 and AS-B634 (left panels). Sphere forming capacities of AS-B145 and AS-B634 transduced with shRNA-TMCC3 (shTM \#A and shTM \#B) vs shRNA-control (shCtrl) after 7 days culture (right panels). Data represent mean \pm SD. $* * p<0.01,{ }^{\#} p<$ 0.001 and $\#^{*} p 0.0001 \quad(n=6, t$-test $)$. Scale bar $=200 \mu \mathrm{m}$. e The representative images of mammospheres and sphere forming capacities of MCF7 overexpressing TMCC3 vs vector control after 7 days culture. Data represent mean $\pm \mathrm{SD} .{ }^{\#} p<0.001(n=6, t$-test $)$. Scale bar $=$ $200 \mu \mathrm{m}$. f, g ALDH activity of TMCC3 silenced AS-B145 and ASB634 (shTM \#A and shTM \#B), as compared with their respective controls. $\mathrm{ALDH}^{+}$subpopulation was determined by flow cytometry and the relative folds of $\mathrm{ALDH}^{+}$cells were calculated from three independent experiments (h). Data represent mean \pm SEM. ${ }^{\# \#} p<$ $0.0001\left(n=3, t\right.$-test). i CD24 $4^{-} \mathrm{CD} 44^{+}$cell population of TMCC3overexpressed MCF7 was determined by flow cytometry, as compared with vector control transduced MCF7. Data represent mean \pm SEM. ${ }^{*} p=0.01(n=6, t$-test $)$.

also determined the mammosphere forming capacity of TMCC3 overexpressing MCF7. As shown in Fig. 1e, greater mammosphere number was observed in TMCC3 overexpressing cells $(33.5 \pm 4.5)$, as compared with the vector control cells $(22.5 \pm 2.9, n=6, p<0.001)$. In addition, $\mathrm{ALDH}^{+}$subpopulation of AS-B145 was reduced from $33.7 \%$ (shControl) to $22.9 \%$ (shTM \#A) and $16.8 \%$ (shTM \#B) upon TMCC3 silencing (Fig. 1f, h), and the relative folds of $\mathrm{ALDH}^{+}$subpopulation of AS-B145 was significantly reduced by shTM \#A and \#B to $0.61 \pm 0.01(n=$ $3, p<0.0001)$ and $0.46 \pm 0.03(n=3, p<0.0001)$ folds, respectively, of shControl. Similarly, $\mathrm{ALDH}^{+}$subpopulation of AS-B634 was significantly reduced by shTM \#A and shTM \#B to $0.68 \pm 0.03(n=3, p<0.0001)$ and $0.49 \pm 0.02$ $(n=3, p<0.0001)$ folds, respectively, of shControl (Fig. $1 \mathrm{~g}, \mathrm{~h})$. On the other hand, upon TMCC 3 overexpression in MCF7, the CD24 ${ }^{-} \mathrm{CD} 44^{+}$subset which has been reported to enrich BCSCs increased from $26.15 \pm 4.38 \%$ to $48.17 \pm$ $5.46 \%(n=6, p=0.01)$ (Fig. 1i) [24]. These findings indicate that TMCC 3 is crucial for sphere formation, ALDH activity, and CSC enrichment, which are important features of BCSCs.

\section{TMCC3 silencing suppresses tumor growth and tumorigenesis in vivo}

We next evaluated the in vivo effects of TMCC 3 silencing on the tumorigenicity of AS-B145 and AS-B634. Control shRNA (shControl) and shRNA-TMCC3 (shTMCC3 \#B) transduced AS-B145 and AS-B634 were injected into mammary fat pads of NSG female mice. Tumor size was monitored weekly for 6-8 weeks, and tumors were harvested for BCSC population examination and Ki67 staining. As shown in Fig. 2, the photographs (a) and weights (b) of tumors from TMCC3 silenced (shTMCC3) AS-B145 and AS-B634 were obviously smaller than control tumors (shControl) $(p<0.01)$. The growth rates of TMCC 3 silenced tumors were also significantly slower than control tumors $(p<0.0001$ for both AS-B145 and AS-B634) (Fig. 2c, d). We also examined the tumor growth of TMCC3 overexpressing MCF7 in vivo. As shown in Fig. 2e, f, TMCC3 overexpressing tumors (Fig. 2f) grew faster than the vector control group (Fig. 2e). Analysis of BCSC cell population $\left(\mathrm{H} 2 \mathrm{k}^{\mathrm{d}-} \mathrm{CD} 24\right.$ ${ }^{-} \mathrm{CD} 44^{+}$) in the TMCC3 silenced AS-B145 tumors showed reduction of BCSCs from $45.8 \%$ in shControl tumors to $15.1 \%$ in shTMCC3 tumors (Fig. 2 g). Furthermore, Ki67 expression was significantly reduced from $24.3 \pm 1.6 \%$ in shControl AS-B634 tumors to $7.2 \pm 1.7 \%$ in TMCC3 silenced tumors $(n=3, p<0.01)$ (Fig. 2h). These findings indicate that TMCC 3 is not only important for tumor growth, but also crucial for the maintenance of BCSC population in vivo.

To determine the tumor-initiating capacity (TIC) of TMCC 3 silenced AS-B145 and AS-B634, serial dilutions of cells were injected into NSG mice. As shown in Table 1, TIC of AS-B145 and AS-B634 decreased from 1:19,707 to $1: 70,682(p=0.014)$ and $1: 59$ to $1: 987 \quad(p<0.001)$, respectively, after TMCC3 knockdown. This provides further evidence for the crucial role of TMCC3 in CSC properties in vivo.

\section{TMCC3 is crucial for tumor metastasis in vitro and in vivo}

The propensity of CSCs for tumor metastasis is well documented $[25,26]$. To delineate the involvement of TMCC 3 in metastasis of breast cancer, we examined the expression of TMCC 3 in metastatic lesions of breast cancer PDXs. Two to three months after injection of BC0145 or BC0634 tumor cells in mammary fat pads of NSG mice, metastatic lesions were observed in ipsilateral lymph nodes and lungs. The lymph nodes and primary tumor of BC0145 PDX were harvested for staining with anti-CD44 and antiTMCC 3 antibodies. As shown in Fig. 3a, 4.1\% and 15.3\% of cells in the BC0145 primary tumor and metastatic lymph node, respectively, were $\mathrm{CD} 44^{+} \mathrm{TMCC}^{+}$. The relative fold of $\mathrm{CD}_{4} 4^{+} \mathrm{TMCC}^{+}$subsets in metastatic lymph node was $2.72 \pm 0.7$, as compared with primary tumor cells (Fig. 3b, $n=5, p=0.04$ ). In addition, the representative IHC staining for TMCC 3 expression of primary tumor, lymph node, and lung-metastatic tissues from one of three BC0634 
Fig. 2 TMCC3 is essential for tumorigenicity in vivo. $\mathbf{a}, \mathbf{b}$ TMCC3 silenced AS-B145, ASB634, and their control cells were implanted into mammary fat pad of NSG female mice and tumor sizes were monitored weekly. The photographs (a) and weights (b) of tumors from control (shControl) and TMCC3 silenced (shTMCC3) AS-B145 and AS-B634. c, d Tumor growth curves of control and TMCC 3 silenced AS-B145 and AS-B634. Data represent mean $\pm \mathrm{SD}(n=5$, AS-B145 and $n=8$, AS-B634). e, f Tumor growth curve of TMCC3overexpressed and vector transduced MCF7. g Effects of TMCC3 silencing on breast cancer stem cell population $\left(\mathrm{H} 2 \mathrm{k}^{\mathrm{d}-} \mathrm{CD} 24^{-} \mathrm{CD} 44^{+}\right)$of AS-

B145 PDX tumor as determined by flow cytometry. $\mathbf{h}$ The expression of Ki67 in shControl and shTMCC 3 transduced ASB634 PDX tumors $(n=3)$. Scale bar $=100 \mu \mathrm{m}$. $* * p<0.01,{ }^{\# \#} p<$ 0.0001 . a

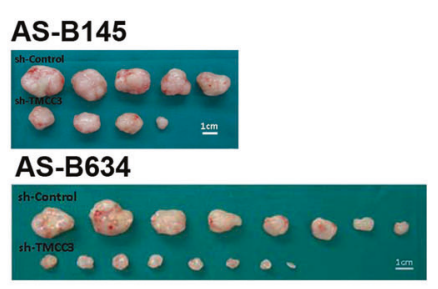

C

AS-B145

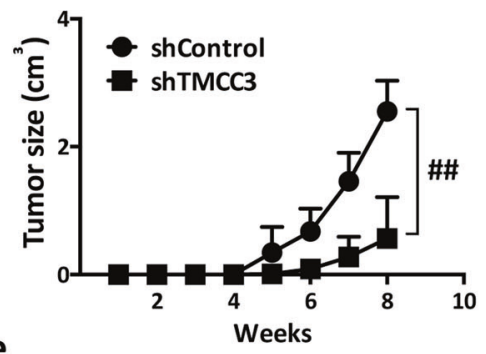

e

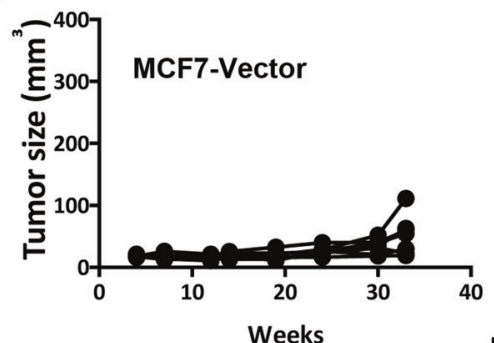

g

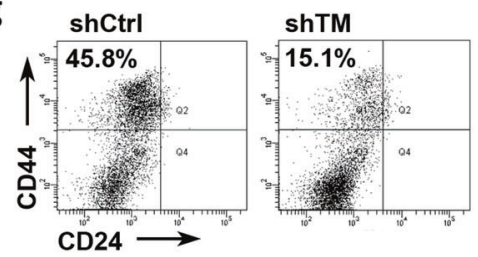

b
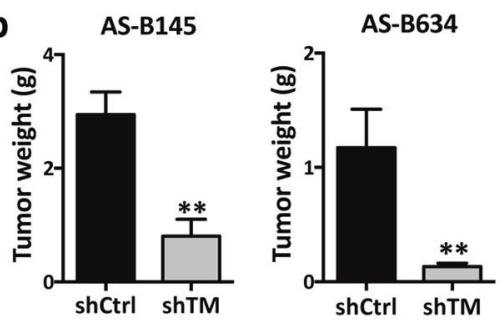

d

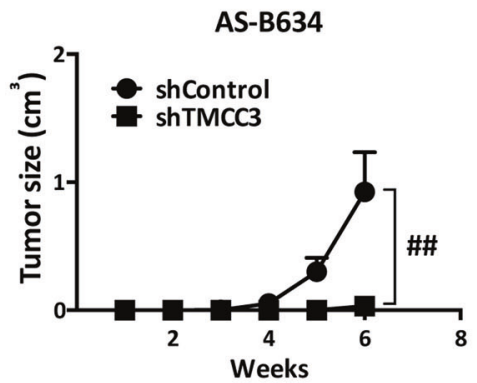

f

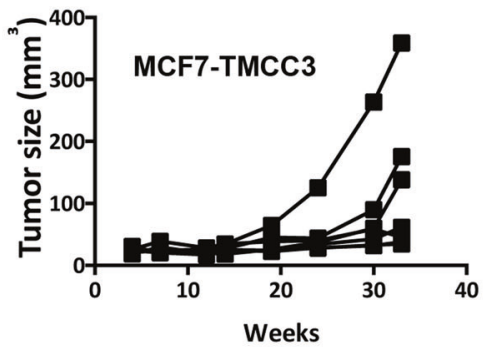

h
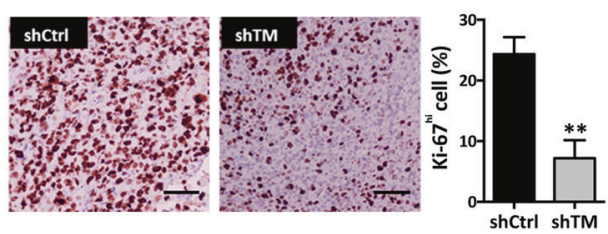

Table 1 TMCC3 silencing of AS-B145 and AS-B634 reduces their tumor-initiating capacities ${ }^{\mathrm{a}}$.

\begin{tabular}{llllllllll}
\hline & & \multicolumn{3}{c}{ (Cell number of injection) } & & Stem cell frequency & $p$ value \\
\cline { 3 - 7 } & & & $10^{5}$ & $5 \times 10^{4}$ & $10^{4}$ & $10^{3}$ & $10^{2}$ & 50 & \\
\hline \multirow{2}{*}{ AS-B145 } & shControl & $5 / 5$ & $5 / 6$ & $3 / 5$ & & & & $1: 19,707$ & 0.014 \\
& shTMCC3 & $4 / 5$ & $3 / 7$ & $1 / 5$ & & & & $1: 70,682$ & $<0.001$ \\
\multirow{4}{*}{ AS-B634 } & shControl & & & $8 / 8$ & $5 / 5$ & $4 / 5$ & $3 / 5$ & $1: 59$ & \\
& shTMCC3 & & & $8 / 8$ & $3 / 5$ & $1 / 5$ & $0 / 5$ & $1: 987$ & $<$ \\
\hline
\end{tabular}

${ }^{\mathrm{a} B C S C}$-enriched population of AS-B145 and AS-B634 were infected with shTMCC3 \#B or shControl and injected into mammary fat pads of NSG mice. bearing mice were shown in Fig. 3c. The histoscores (hscore) of TMCC 3 expression in tumors as shown in Fig. 3d demonstrated highest TMCC 3 expression in lung lesions followed by lymph node and then primary tumors $(n=3, p$ $<0.05$ ). These findings suggest that TMCC 3 may contribute to tumor metastasis.

To examine the role of TMCC 3 in tumor metastasis, we next determined whether TMCC 3 silencing suppresses the migration of TMCC 3 expressing BCSCs in migration assay.
TMCC3 silencing of AS-B145 and AS-B634 significantly reduced the migrated cell numbers to $1.19 \pm 0.03$ and $1.02 \pm 0.03(\times 1000)$ cells/well, respectively, as compared to $2.72 \pm 0.26$ and $3.03 \pm 0.35(\times 1000)$ cells/well, respectively, in control cells (Fig. 3e, f, $n=3, p<0.01$ ).

To provide further evidence supporting the notion that TMCC 3 is important for metastasis, we examined the impact of TMCC3 silencing on the metastatic frequency of ASB634. After injection of AS-B634, primary tumor, lymph 
Fig. 3 TMCC3 contributes to tumor metastasis in vitro and in vivo. a FACS analysis of TMCC 3 expression in permeabilized BCSC $\left(\mathrm{CD} 44^{+}\right)$ harvested from primary tumor (Tumor) and metastatic lymph node (LN-metastasis) of BC0145 PDX tumor. b Percent of $\mathrm{CD}_{4} 4^{+} \mathrm{TMCC}^{+}$cell population in metastatic lymph node was normalized to that in primary tumor to show the relative folds. ${ }^{*} p<0.05(n=5$, $t$-test). c The representative IHC stainings of primary tumor, lymph node, and lung-metastatic tissues from one of three BC0634 bearing mice. Scale bar $=100 \mu \mathrm{m}$. d The histoscores (hscore) of TMCC 3 expression in tumors of three mice were calculated. $* p<0.05, * * * p<$ 0.001 ( $n=3, t$-test). e, f The numbers of migrated cells in each group were determined in trans-well migration assay. $* * p$ $<0.01$ ( $n=3$, $t$-test). Scale bar $=100 \mu \mathrm{m}$. g Metastatic frequency of TMCC 3 silenced AS-B634. $2 \times 10^{4}$ shTMCC3 or shControl transduced AS-B634 were implanted into mammary fat pad of NSG mice $(n=4)$. Tumor, lymph node, and lung of tumor bearing mice were harvested at 3 months after implantation. a

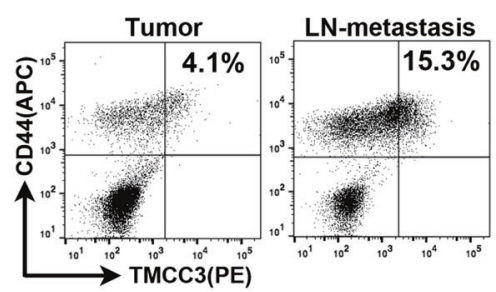

C

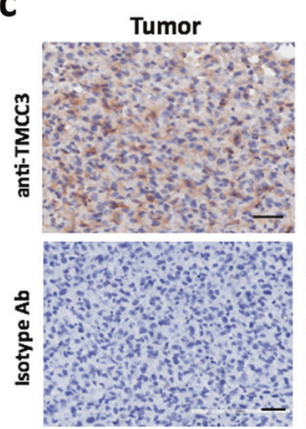

e

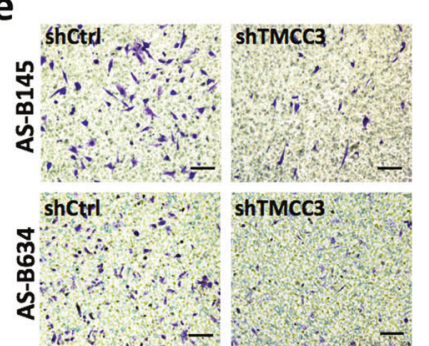

f b

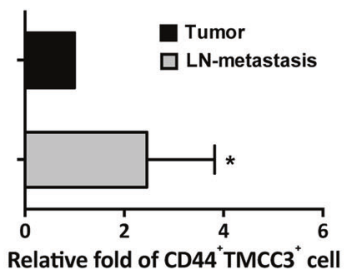

d
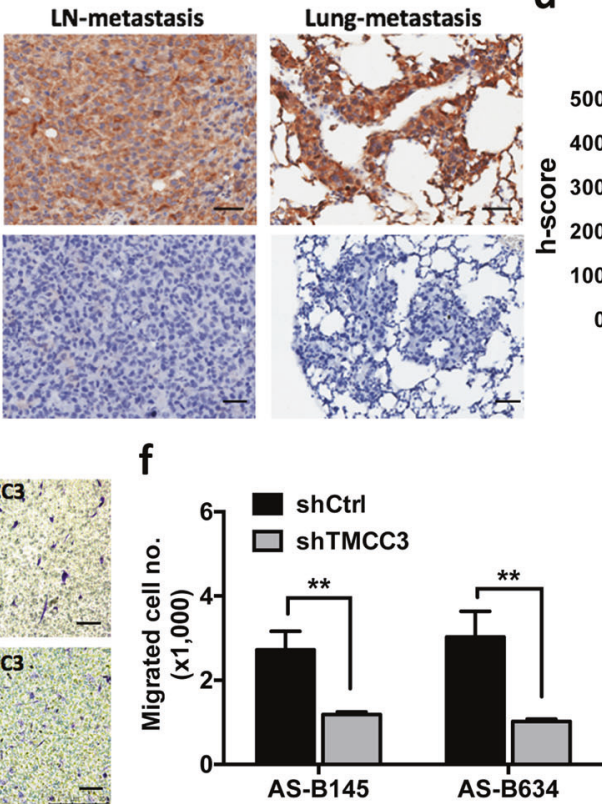

\section{g}

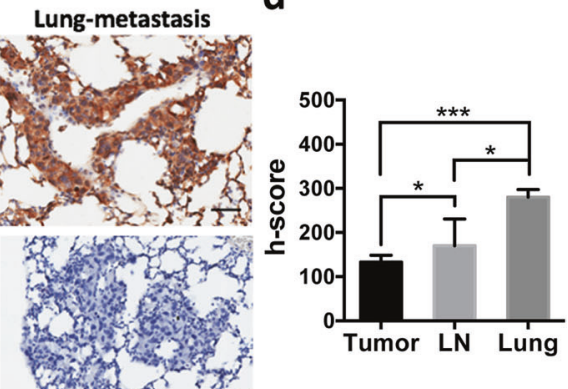

node, and lung of tumor bearing mice were harvested at 3 months. As shown in Fig. 3g, we found TMCC 3 silencing abolished metastasis in vivo. The lymph node and lungmetastatic frequency were $75 \%(3 / 4)$ and $50 \%$ (2/4), respectively, in control group, but none in TMCC 3 knockdown. These findings support the participation of TMCC 3 in tumor metastasis in vitro and in vivo.

\section{TMCC3 is involved in AKT activation in BCSCs}

It is well known that AKT plays important roles in the center of diverse signaling cascades and essential to stem cell activation, CSC survival, and self-renewal [27-32]. Previously, we demonstrated that IGF-1R/PI3K/AKT/ mTOR pathway was important for BCSC maintenance [14]. To examine a possible linkage of TMCC3 to AKT signaling, we determined AKT phosphorylation in TMCC3 silenced AS-B634. As shown in Fig. 4a, TMCC3 knockdown by shTM \#A and \#B decreased the relative folds of $\mathrm{pAKT}^{\mathrm{S} 473} / \mathrm{AKT}$ to 0.4 and 0.2 , and $\mathrm{pAKT}^{\mathrm{T} 308}$ / AKT to 0.6 and 0.3 in AS-B634, respectively. On the other hand, relative folds of $\mathrm{pAKT}^{\mathrm{S} 473}$ and $\mathrm{pAKT}^{\mathrm{T} 308}$ increased to 1.3 and 1.5 in TMCC3 overexpressing MCF7 upon insulin stimulation, respectively (Fig. 4b). These findings suggest that AKT activation may contribute to the critical role of TMCC3 in maintenance of BCSC properties. We further examined the possibility that TMCC 3 may enhance AKT phosphorylation via activation of PI3K/PDK1, the up-stream regulators of AKT. As shown in Fig. 4a, b and Supplementary Fig. S3, the phosphorylation of regulatory subunit p85 (p-p85/p85) of PI3K and PDK1 (pPDK/PDK) did not decrease upon TMCC3 silencing in AS-B634 or increase in TMCC3 overexpressing MCF7. In addition, TMCC3 overexpression in MCF7 did not enhance membrane translocation of catalytic subunit p110- $\alpha$ of PI3K (Fig. 4c). These findings suggested that TMCC3-induced AKT activation likely does not involve PI3K or PDK1 phosphorylation. 

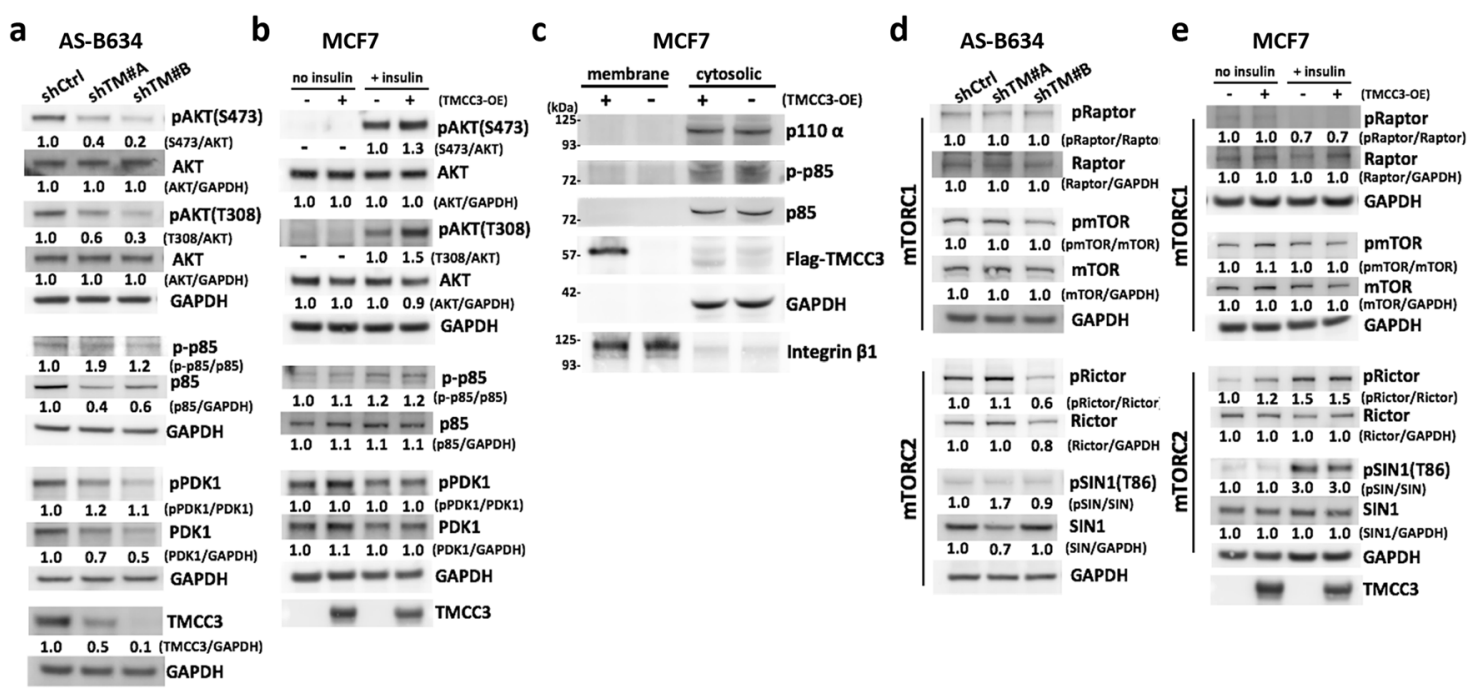

Fig. 4 TMCC3-induced AKT activation likely does not involve PI3K/PDK1/mTORC1 or mTORC2 pathway. AS-B634 cells were transduced with shRNA-TMCC3 \#A, \#B, or shRNA-control for 3 days. MCF7 cells were transiently transfected with TMCC3 in the presence $(+)$ or absence $(-)$ of $10 \mu \mathrm{g} / \mathrm{ml}$ human insulin for $5 \mathrm{~min}$ after $12 \mathrm{~h}$ serum starvation. Levels of phosphorylated AKT (S473 and T308), p85 of PI3K and PDK1, and total protein in TMCC3 silenced AS-B634 (a) and TMCC3 overexpressing MCF7 (b), were determined

by western blotting. Relative intensity was quantified and normalized to non-phospho protein or GAPDH. c Subunit p85 phosphorylation and $\mathrm{p} 110-\alpha$ membrane translocation were determined in TMCC3 overexpressing MCF7 cells. Integrin $\beta 1$ and GAPDH served as membrane and cytoplasmic markers, respectively. d, e mTORC1 (Raptor and mTOR) and mTORC2 (Rictor and SIN1) activation were assessed in TMCC3 silenced AS-B634 and TMCC3 overexpressing MCF7 cells.

Recent studies have identified that mTOR complex 1 (mTORC1) and mTORC2 activation correlate to AKT phosphorylation [33-35]. To explore the consequence of TMCC3-induced AKT activation, we examined mTORC1 and mTORC2 phosphorylation upon TMCC3 knockdown or overexpression. As shown in Fig. 4d, e, there were no alterations in the activation of mTORC1 (pRaptor/Raptor and $\mathrm{p}-\mathrm{mTOR} / \mathrm{mTOR}$ ) and mTORC2 (pRictor/Rictor and pSIN1/SIN1) in TMCC3 silenced or overexpressing cells. However, the use of phosphoproteins/total proteins ratios to reflect activation status were affected by reduction of total p85 and PDK1 proteins in shTM\#A and shTM\#B transduced AS-B634 (Fig. 4a), and total Rictor and SIN1 proteins in one of shRNA-TMCC3 transduced cells (Fig. 4d). It should be noted that TMCC3 silencing reduced cell growth (Supplementary Fig. S4), which may explain the decrease in these signaling proteins. Taken together, these findings suggested that PI3K, PDK1, mTORC1, or mTORC2 signalings are most likely not involved in TMCC3-induced AKT activation.

\section{1-153 a.a. domain of TMCC3 contributes to interaction and activation of AKT}

In order to ascertain the regulation of TMCC3 on AKT activation, we pulled down the endogenous TMCC 3 in ASB634 with anti-TMCC3 antibody. As shown in Fig. 5a, AKT was detected in the immunoprecipitation. To confirm the interaction of TMCC3 and AKT, we constructed

flag-tagged TMCC3 and HA-tagged AKT for coimmunoprecipitation assay. As shown in Fig. 5b, c, HAtagged AKT was pulled down with flag-tagged TMCC3 using anti-flag antibody, and vice versa.

To decipher the AKT-interacting domain of TMCC3, we constructed flag-tagged full-length (1-477), 1-153, 1-282, 154-282, and 283-416 amino acid (a.a.) domains of TMCC3 for immunoprecipitation assay (Fig. 5d). 293T cells were co-transfected with flag-tagged TMCC3 (full-length or truncated protein) and HA-tagged AKT. After flag protein pull-down using anti-flag antibody, the immunoprecipitates were examined for HA-AKT proteins with anti-HA antibody. As shown in Fig. 5e, AKT was pulled down together with 1-153, 1-282 a.a. domains and full-length TMCC3, but not with 283-416 a.a. domain. However, the expression of 154-282 a.a. domain of TMCC3 was too low to delineate whether it interacted with AKT or not. To provide evidence for direct interaction between TMCC 3 and AKT in a cellfree system, luminex immunosandwich assay was performed as illustrated in Fig. $5 f$ [36]. Recombinant 1-158 a.a. domain of TMCC 3 protein was generated and coupled onto Luminex beads, which were then incubated with various concentrations of recombinant AKT. After washing, AKT interacting with bead-bound 1-158 a.a. domain of TMCC3 were recognized by the fluorescence labeled anti-AKT antibody. As shown in Fig. 5g, AKT can bind directly with 1-158 a.a. domain of TMCC3 protein in vitro in a concentration dependent manner, with $\mathrm{KD}$ of $1.67 \pm 0.43 \mathrm{nM}$. On the other hand, AKT did not bind to bead-bound control 


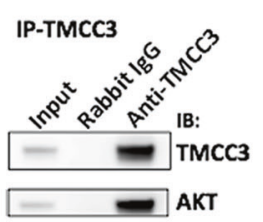

d b

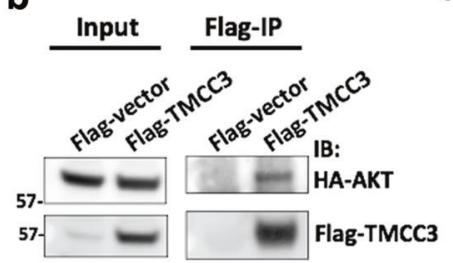

C

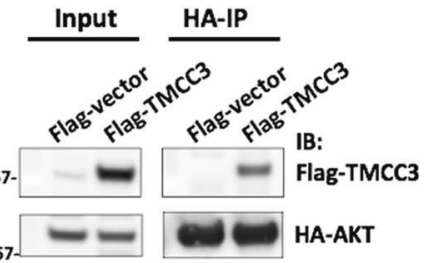

e

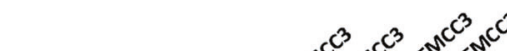

Domain mapping of TMCC3

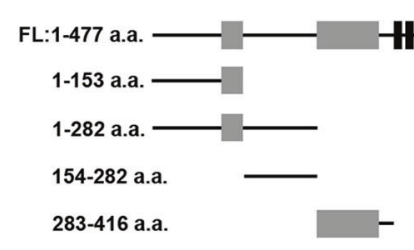

H HA-AKT: +
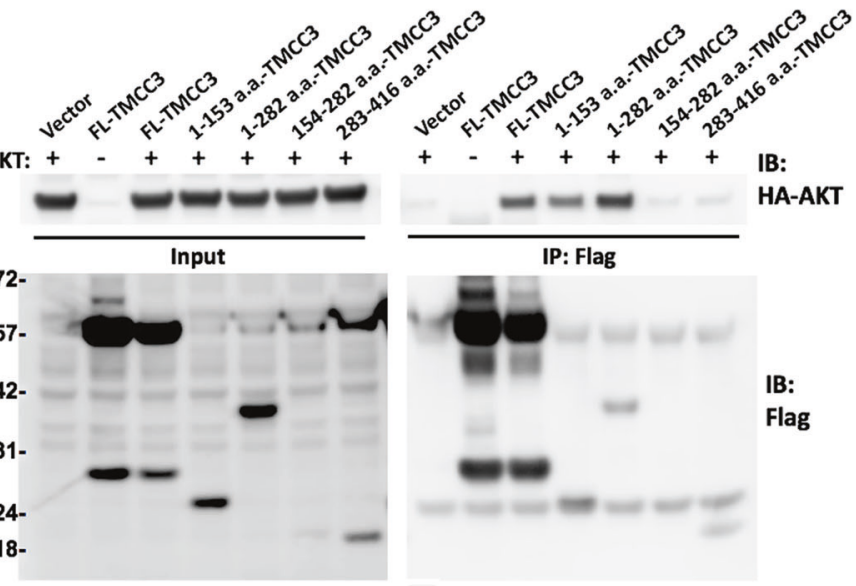

f

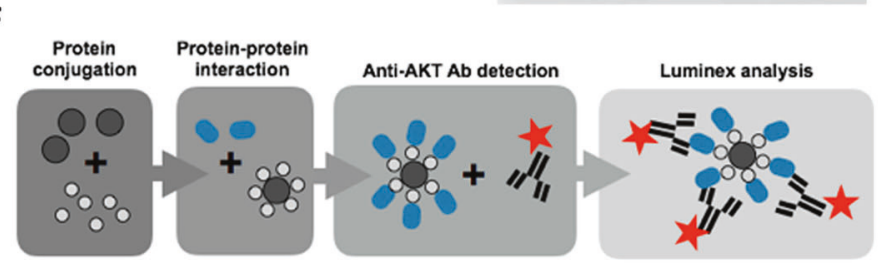

$O$ : recombinant 1-158 aa of TMCC3

: Luminex xMAP beads
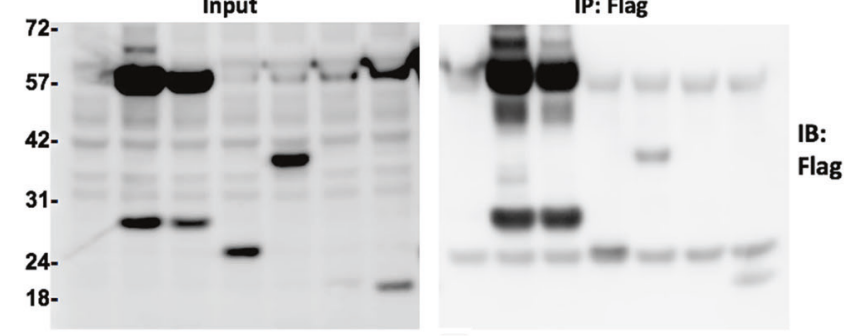

g

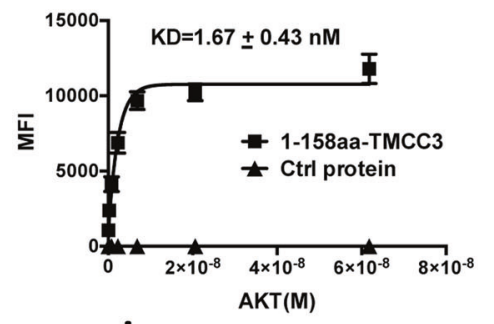

h

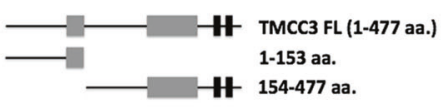

i

j

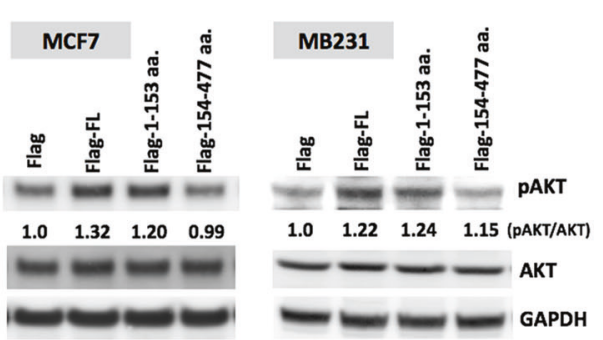

Fig. 5 1-153 a.a. domain of TMCC3 directly interacts with AKT and enhances AKT activation. a Presence of AKT in immunoprecipitate of TMCC3 from AS-B634 using anti-TMCC3 antibody. b, c MDA-MB231 cells transfected with flag-tagged full-length (FL)TMCC 3 and HA-tagged AKT were subject to co-immunoprecipitation assay using anti-flag antibody (b) or anti-HA antibody (c), followed by immunoblotting. d Diagram of full-length TMCC 3 and truncated forms of TMCC3 containing 1-153 a.a., 1-282 a.a., 154-282 a.a., 283416 a.a. e Co-immunoprecipitation analysis of flag-tagged TMCC3 with HA-tagged AKT proteins. 293T cells were transfected with a plasmid encoding HA-tagged AKT and another plasmid encoding flag tag alone or various flag-tagged TMCC3 forms: full-length (FL), 1-153 a.a., 1-282 a.a., 154-282 a.a., or 283-416 a.a. f Flowchart of luminex
MCF7
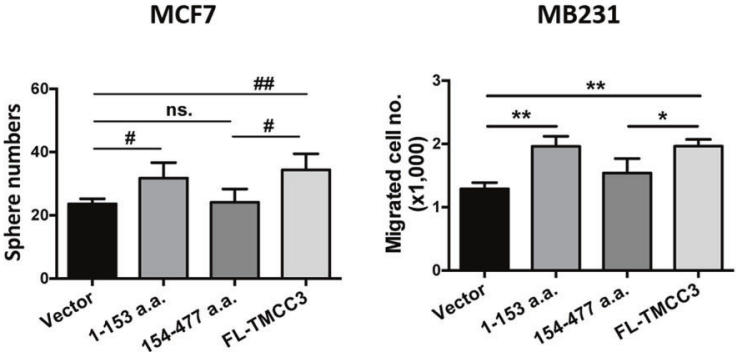

immunosandwich assay. Detailed procedures were described in "Materials and methods". g Mean fluorescence intensities (MFI) showed AKT bound to beads coupled with 1-158 a.a. domain of TMCC3 proteins $(n=4)$. h Levels of pAKT ${ }^{\mathrm{S} 473}$ and total AKT were examined in flag-tagged full-length (Flag-FL), 1-153 a.a.- or 154-477 a.a.-truncated TMCC3 transfected MCF7 and MDA-MB231 (MB231) by western blot analysis. Mammosphere forming capacities (i) and migration abilities (j) were assessed in cells transfected with flagtagged full-length (Flag-FL), 1-153 a.a.- or 154-477 a.a.-truncated TMCC 3. i Sphere numbers were calculated after culture for 7 days. ${ }^{\#} p<0.001,{ }^{\# \#} p<0.0001(n=8, t$-test $) . \mathbf{j}$ The numbers of migrated cells in each group were determined in trans-well migration assay. $* p<0.05, * * p<0.01(n=3, t$-test $)$. 


\section{a}

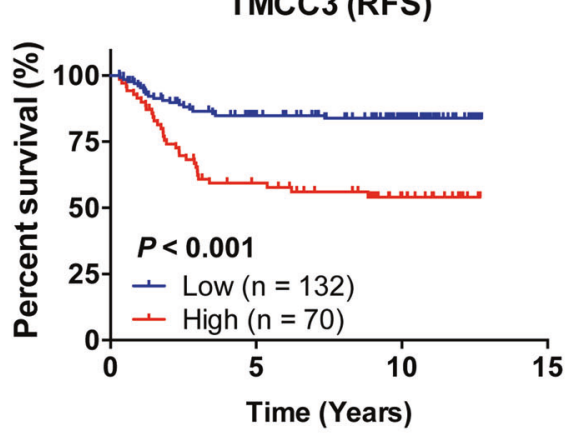

C

Stage1-2 (RFS)

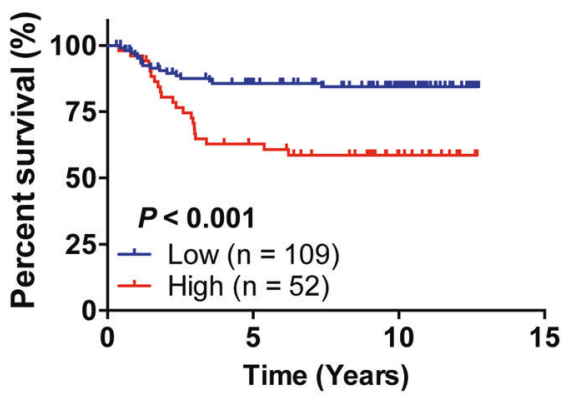

e

Stage1-2 (Luminal subtype)

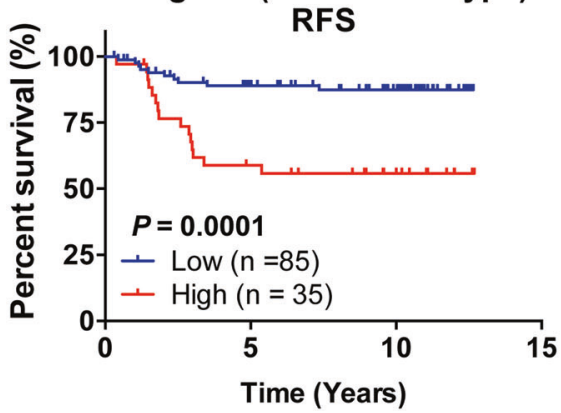

Fig. 6 High expression of $T M C C 3$ correlates with poor clinical outcome of breast cancer patients. a, b The mRNA levels of TMCC 3 in tumor specimens of 202 patients with breast cancer were analyzed by qRT-PCR. Kaplan-Meier plots of relapse-free survival (RFS) and overall survival (OS) for patients in relation to TMCC3 mRNA levels, and using Youden index to determine the optimal cutoff values defining high (red) and low (blue) expression groups. High and low expression of TMCC3 in relation to RSF (a) and OS (b) were shown in tumor tissue $(n=202)$ with breast cancer. High and low expression of $T M C C 3$ in relation to RSF (c) and OS (d) were shown in stages 1-2

protein (Puf-A) [37]. These findings support the notion that 1-158 a.a. domain of TMCC3 directly interacted with AKT without other accessory proteins.

To delineate whether interaction of TMCC3 with AKT contributes to TMCC3-induced AKT activation, we generated TMCC 3 constructs containing truncation of 1-153 a.a. or 154-477 a.a.. MCF7 and MDA-MB231 (MB231) cells were transfected with flag-tagged full-length, 1-153 a.a.-, 154-477 a.a.-truncated TMCC3 or vector control b

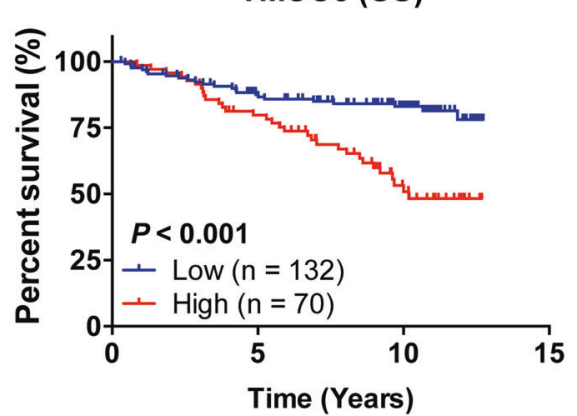

d

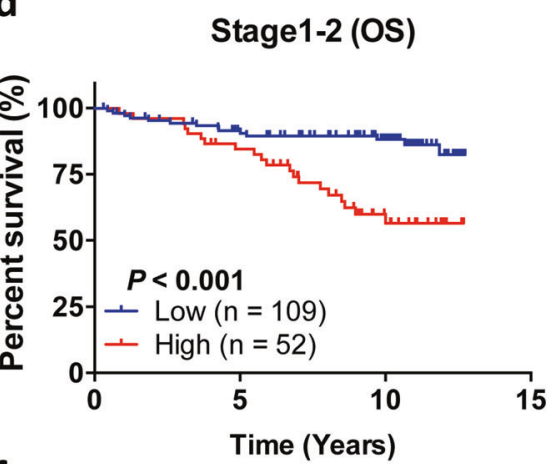

f

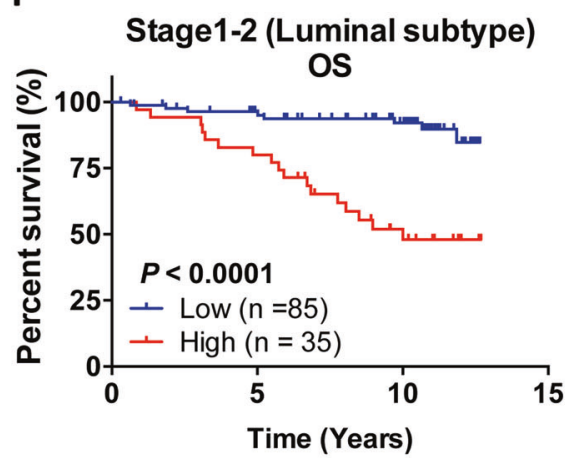

breast cancer tumor tissue ( $n=161$ for stages $1-2$ breast cancers). In stages 1-2 luminal A + B breast cancers, high and low expression of $T M C C 3$ in relation to RSF (e) and OS (f) were shown in tumor tissue ( $n=120$ for stages $1-2$ luminal A + B breast cancers). Survival curves were plotted with Kaplan-Meier method, by the log-rank test applied for comparison. The Cox proportional-hazards regression model was employed to evaluate the independent prognostic factors. The statistical analyses were performed with Prism 5.0 (GraphPad Software, La Jolla) and SPSS ver. 22.0 (IBM, Armonk) software.

(Supplementary Fig. S5), and pAKT ${ }^{\mathrm{S} 473}$ and total AKT were determined. As shown in Fig. 5h, pAKT ${ }^{\mathrm{S} 473}$ was upregulated to 1.32 and 1.20 folds of vector control in MCF7 transfected with full-length or 1-153 a.a.-truncated TMCC3, respectively. But no change in cell transfected with 154-477 a.a.truncated TMCC3. Similar results were also observed in MB231 cells. pAKT ${ }^{\mathrm{S} 473}$ was increased to 1.22 and 1.24 folds of vector control in cells transfected with full-length or 1-153 a.a.-truncated TMCC3, respectively. These findings 
suggest that 1-153 a.a. domain of TMCC3 which interacted directly with AKT is crucial for AKT activation. In addition, we further examined the contribution of AKT-interacting region of TMCC3 to mammosphere formation and migration ability. MCF7 and MDA-MB231 were transfected with flag-tagged full-length, 1-153 a.a.-, 154-477 a.a.-truncated TMCC3 or vector control. As shown in Fig. 5i, mammosphere formation was significantly enhanced in cells transfected with 1-153 a.a. domain $(31.75 \pm 1.74, n=8$, $p<0.001)$ or FL-TMCC3 $(34.38 \pm 1.80, p<0.0001)$, as compared with vector control cells $(23.63 \pm 0.57)$, but not in 154-477 a.a. domain of TMCC3 expressing cells $(24.13 \pm$ 1.48). Next, we examined the migration ability of truncated or full-length TMCC3 expressing cells. As shown in Fig. 5j, the migration capacity was significantly improved in cells expressing 1-153 a.a. domain or FL-TMCC3, as compared with control cells $(n=3, p<0.01$ and $p<0.01$, respectively), but not in 154-477 a.a. domain of TMCC3 transfected cells. Our findings support that AKT-interacting domain (1-153 a.a.) of TMCC3 could enhance AKT activation and contribute to BCSC properties.

\section{Expression of TMCC3 mRNA in breast cancer tissues}

To evaluate the clinical relevance of TMCC3 expression, we examined the mRNA levels of TMCC3 in tumor specimens of 202 patients with breast cancer by qRT-PCR. Clinical characteristics and demographic information of these patients were summarized in Supplementary Table S2. The mean age was $54.4 \pm 12.2$ (range: $30-89$ ). The median follow-up time was 9.55 years (range: 0.30-12.7 years). The relationship between TMCC3 mRNA expression and clinical pathological variables in breast cancer was presented in Supplementary Table S3. Among 202 patients, 51 patients (25.7\%) had disease recurrence. Using Youden index to determine the optimal cutoff values defining high and low expression groups, we found that patients with high expression level of tumor TMCC3 mRNA were at greater risk than those with low expression level for lymph nodes involvement $(p=0.04$, OR: $1.89,95 \%$ CI: $1.06-3.42)$, relapse $(p<0.001$, OR: 4.45 , 95\% CI: $2.27-8.69)$, and death $(p<0.001$, OR: $3.77,95 \%$ CI: 1.96-7.23) (Supplementary Table S3).

\section{Association of TMCC3 expression levels with survival}

The relapse-free survival (RFS) and overall survival (OS) of breast cancer patients as analyzed by the Kaplan-Meier method, and a log-rank test showed that patients with low expression level of TMCC3 in tumor had significantly greater RFS $(p<0.001)$ and OS $(p<0.001)$ than patients with high expression levels (Fig. 6a, b). We then analyzed the potential prognostic value of TMCC3 expression in 161 patients with early-stage disease (stages 1-2). As shown in
Fig. 6c, d, early-stage patients with low expression of TMCC3 in tumor part had significantly greater RFS $(p<0.001)$ and OS $(p<0.001)$ than patients with high expression levels. The survival benefit of patients with low expression of TMCC3 was even more striking for earlystage luminal A and B patients ( $p=0.0001$ for RFS, $p<0.0001$ for OS) (Fig. 6e, f). These results demonstrated the adverse impacts of high expression of TMCC3 on clinical outcome of breast cancer, especially for those with early-stage luminal subtypes.

\section{Higher expression level of TMCC3 is an independent prognostic factor for breast cancer}

To evaluate the potential value of TMCC3 expression levels for predicting RFS and OS of breast cancer, univariate Cox proportional hazard regression analyses were conducted. The results indicated that RFS correlated with patients aged $\geq 50$ years (HR: 2.74, 95\% CI: $1.43-5.24, p=0.002$ ) and Grade III (HR: 1.89, 95\% CI: $1.04-3.46, p=0.038$ ), and higher expression of TMCC3 in tumor (HR: 3.32, 95\% CI: $1.89-5.83, p=0.001$ ) (Table 2). In addition, OS correlated with the patients aged $\geq 50$ years (HR: 2.66, 95\% CI: 1.42-4.96, $p=0.002$ ), Grade III (HR: 1.89, 95\% CI: $1.06-3.40, p=0.032$ ), stages III + IV (HR: $2.81,95 \%$ CI: $1.62-4.89, p=0.001)$ and higher expression of TMCC3 in tumor (HR: 2.92, 95\% CI: 1.69-5.02, $p=0.001$ ) (Table 2). Next, to identify the independent variables associated with poor RFS or OS, we selected those important covariates that showed statistical significance in the univariate analysis for multivariable Cox regression analysis in a stepwise manner. As shown in Table 2, age was independent risk factors for RFS. And age and stage were independent risk factors for OS. Notably, the expression level of tumor TMCC3 was an independent risk factor for RFS (HR: 2.81, 95\% CI: $1.45-5.45, p=0.002)$ and OS (HR: $2.28,95 \% \mathrm{CI}$ : $1.31-3.96, p=0.004)$. These data indicate that TMCC3 expression in tumor tissue is an important and independent predictor for RFS and OS in breast cancer patients.

\section{Data mining confirms poor prognostic impact of high expression of TMCC 3 in breast cancer and other cancers}

Using an online DNA microarray database at the ONCOMINE website, we found higher mRNA level of TMCC3 in cancer part than normal tissue in cervical cancer, prostate cancer, pancreatic cancer, lung cancer, glioblastoma, skin cancer, hepatoma, and thyroid gland papillary carcinoma (Supplementary Fig. S6a). Using Kaplan-Meier Plotter website to evaluate the clinical significance of TMCC3 in cancer progression, we showed that higher expression of TMCC3 correlated with poor OS of patients with ovarian, 
Table 2 Association of risk factors with recurrence and survival of breast cancer in univariate and multivariable analysis.

\begin{tabular}{|c|c|c|c|c|c|c|c|c|c|c|c|c|}
\hline \multirow[t]{3}{*}{ Variables } & \multicolumn{6}{|l|}{ RFS } & \multicolumn{6}{|l|}{ OS } \\
\hline & \multicolumn{3}{|c|}{ Univariate analysis } & \multicolumn{3}{|c|}{ Multivariable analysis } & \multicolumn{3}{|c|}{ Univariate analysis } & \multicolumn{3}{|c|}{ Multivariable analysis } \\
\hline & HR & $95 \% \mathrm{CI}$ & $p$ value & HR & $95 \% \mathrm{CI}$ & $p$ value & HR & $95 \% \mathrm{CI}$ & $p$ value & $\mathrm{HR}$ & $95 \% \mathrm{CI}$ & $p$ value \\
\hline Age: $\geq 50$ vs $<50$ & 2.74 & $1.43-5.24$ & 0.002 & 2.54 & $1.32-4.89$ & 0.005 & 2.66 & $1.42-4.96$ & 0.002 & 2.35 & $1.25-4.43$ & 0.008 \\
\hline Grade: III vs I + II & 1.89 & $1.04-3.46$ & 0.038 & & & & 1.89 & $1.06-3.40$ & 0.032 & & & \\
\hline Stage: III + IV vs I + II & 1.74 & $0.94-3.23$ & 0.08 & & & & 2.81 & $1.62-4.89$ & 0.001 & 2.38 & $1.36-4.17$ & 0.002 \\
\hline ER: $(+)$ vs $(-)$ & 0.63 & $0.36-1.08$ & 0.09 & & & & 1.13 & $0.65-1.97$ & 0.66 & & & \\
\hline PR: $(+)$ vs $(-)$ & 0.59 & $0.34-1.03$ & 0.06 & & & & 0.79 & $0.45-1.38$ & 0.40 & & & \\
\hline HER2: $(+)$ vs $(-)$ & 0.86 & $0.49-1.50$ & 0.59 & & & & 0.88 & $0.51-1.51$ & 0.64 & & & \\
\hline TMCC3 Tumor: high vs low & 3.32 & $1.89-5.83$ & 0.001 & 2.81 & $1.45-5.45$ & 0.002 & 2.92 & $1.69-5.02$ & 0.001 & 2.28 & $1.31-3.96$ & 0.004 \\
\hline
\end{tabular}

$R F S$ relapse-free survival, $O S$ overall survival, $H R$ hazard ratio, $C I$ confidence interval.

Bold values indicate statistical significance $p<0.05$.

lung, and gastric cancer (Supplementary Fig. S6b). These findings lend further support for the crucial roles of TMCC3 in cancer progression, and for developing strategies to target TMCC3 for cancer therapy.

\section{Discussion}

In our previous comparative phosphoproteomic analysis of BCSCs and non-BCSCs, we found a function unknown protein, TMCC3 to be expressed at higher level in BCSCs than non-BCSCs [15]. Here, we demonstrated that TMCC3 is crucial for maintaining the characteristics of BCSCs, including self-renewal, differentiation, metastasis, and tumorigenesis. In addition, we showed that TMCC 3 could enhance AKT phosphorylation, which is known to promote BCSC properties.

TMCC3 was reported as an ER-anchored protein and localized at the three-way junctions of tubular ER network $[20,38]$. In mouse developing embryos, TMCC3 was expressed in the mesenchyme of developing tongue, hind brain forming tissues and lung, based on in situhybridization analysis [20]. However, the biological functions of TMCC 3 remain enigmatic. Our study has provided the first evidence for the crucial role of TMCC 3 in sustaining BCSCs properties as reflected by diminished mammosphere forming capacity, ALDH activity, in vivo metastasis and tumorigenicity upon TMCC 3 silencing. In addition, we demonstrated that TMCC 3 promoted AKT activation, and TMCC3 directly interacted with AKT through 1-153 a.a. fragment. This AKT-interacting domain of TMCC3 is crucial for TMCC3-induced AKT activation, mammosphere formation, and metastasis.

Dysregulation of AKT pathway is frequently observed in many type of cancers, including breast cancer, and associated with poor outcome [39, 40]. AKT activation contributes to self-renewal and differentiation of normal mammary stem cell and BCSCs [41, 42]. Mechanistically, we found TMCC 3 contributes to self-renewal and metastasis by the direct binding and activation of AKT via 1-153 a.a. domain of TMCC 3 . In previous reports, TMCC 3 was found to bind with 14-3-3 in HEK 293 cells [20]. In our mass spectrometric analysis, we did not find 14-3-3 family proteins as interacting partners in the immunoprecipitates of TMCC 3 in MCF7 cells. This suggests that the interacting proteins and regulatory mechanisms of TMCC 3 might vary according to the types of cells. Based on our cell-free biochemical studies, recombinant 1-158 a.a. domain of TMCC3 can directly interact with AKT without any accessory proteins. However, whether any other molecular complexes are recruited or involved in TMCC3-induced AKT activation remains to be determined.

AKT plays a center role of tumorigenesis. Upon activation, p110 PIK3CA phosphorylates phosphatidylinositol $(3,4)$-bisphosphate (PIP2) to form phosphatidylinositol (3,4,5)-trisphosphate (PIP3). Binding of AKT to PIP3 leads to AKT translocation from the cytoplasm to the plasma membrane in close proximity to PDK1, which phosphorylates AKT at T308 [43, 44]. The full activation of AKT requires $\mathrm{AKT}$ to be phosphorylated at $\mathrm{S} 473$ by mTORC2 [34]. Although, TMCC3 activates AKT phosphorylation at both T308 and S473, we have provided experimental evidence that PI3K, PDK1, mTORC1, or mTORC2 pathways are most likely not involved in TMCC3-induced AKT activation. Although AKT is known to be activated by PI3K/ PDK1, several reports show that AKT can be activated independent of PI3K/PDK1 [45-49]. In addition, many studies have demonstrated the existence of complex crosstalks between AKT and multiple cell signaling cascades, which can further promote cancer progression and influence drug sensitivity [50]. Whether TMCC3 participates in such crosstalks awaits further investigation.

Through analysis of proteomics profiling of colorectal cancer and chronic lymphocytic leukemia, TMCC 3 protein 
level was found to be greater in cancer parts than their normal counterparts $[51,52]$. However, the clinical relevance of TMCC 3 was not addressed. Our study is the first to demonstrate the prognostic significance of TMCC 3 in breast cancer. More importantly, we found that high TMCC3 expression adversely impacted clinical outcome in earlystage luminal breast cancer. Until now, Oncotype DX, MammaPrint, and PAM50 are the only validated biomarker assays for early-stage luminal breast cancer [53]. However, these tests are quite costly and complex. The expression level of TMCC3, if confirmed to be highly prognostic in future study, will make TMCC3 as a simple and valuable biomarker for early-stage luminal breast cancer.

In view of the adverse impact of high expression of TMCC 3 on clinical outcome of breast cancer, which was corroborated by similar prognostic significance of TMCC3 in ovarian, lung and gastric cancers by data mining, and the negligible expression of TMCC3 mRNA in human vital organs (heart, lung, liver, and kidney) based on microarray database at the ONCOMINE, TMCC 3 an ideal target for the design of therapeutics to eradicate CSCs in the future.

In conclusion, we provide the first evidence supporting that TMCC3 plays important roles in maintaining BCSCs features, tumor metastasis, and tumorigenicity. TMCC3 enhances AKT activation through direct interaction with AKT. Clinically, high TMCC 3 expression is an independent poor prognostic factor in breast cancer, including earlystage breast cancer. These findings support future pursuit of TMCC 3 as a target for BCSCs-directed therapeutic agents.

\section{Materials and methods}

\section{Cell culture}

See Supplementary information for details.

\section{Clinical specimens}

See Supplementary information for details.

\section{RNA extraction and qRT-PCR analysis}

See Supplementary information for details.

\section{Mammosphere formation assay}

See Supplementary information for details.

\section{Western blotting and immunoprecipitations}

See Supplementary information for details.
Aldefluor assay, FACS analysis, and cell sorting

See Supplementary information for details.

Immunohistochemistry assay

See Supplementary information for details.

\section{Cell migration assay}

See Supplementary information for details.

\section{Xenograft tumorigenicity}

See Supplementary information for details.

\section{Lentiviral vector production and XCELLigence analysis}

See Supplementary information for details.

\section{Recombinant protein production and luminex-based protein-protein interaction assay}

See Supplementary information for details.

\section{DNA construct and DNA transfection}

See Supplementary information for details.

\section{Statistical analysis}

See Supplementary information for details.

Acknowledgements We are grateful to the National RNAi Core Facility at Academia Sinica in Taiwan, for providing shRNA reagents and related services. We also thank Cancer Registry Group of TriService General Hospital for providing clinical data.

Author contributions YHW and ALY developed the study concept design and writing of manuscript. YH, YTC, MWK, YJL, SHW, and JCW performed parts of the experiments. THH and JTH analyzed the clinical data. SCC and JCY provisioned clinical specimens. YHW performed the most of experiments, collected the data, analyzed, and interpreted the data under the supervision of JY and ALY.

Funding This study was supported by grants to ALY from the Ministry of Science and Technology in Taiwan (MOST106-3114-B-182A001, MOST106-2811-B-182A-017, MOST107-2321-B-182A-005, MOST107-2811-B182A-504, MOST108-2321-B-182A-004, MOST 108-2811-B-182A-505, MOST109-2321-B-182A-005, MOST1092811-B-182A-501), Taiwan Biosignature for Breast Cancer (BP004), and Chang Gung Medical Foundation (OMRPG3C0011-16). Grant from Chang Gung Medical Foundation (CMRPG3F0973 to JY; CMRPG3G1531-1533 to JTH) and Chang Gung Postdoctoral Fellowship Award to YHW. 


\section{Compliance with ethical standards}

Conflict of interest YHW, JY, and ALY are holders of a patent for "Methods for suppressing cancer by inhibition of TMCC3" US patent 9,447,193. The remaining authors (YTC, THH, JTH, MWK, SHW, YH, YJL, SCC, JCY, and JCW) declare no competing financial interests.

Publisher's note Springer Nature remains neutral with regard to jurisdictional claims in published maps and institutional affiliations.

Open Access This article is licensed under a Creative Commons Attribution 4.0 International License, which permits use, sharing, adaptation, distribution and reproduction in any medium or format, as long as you give appropriate credit to the original author(s) and the source, provide a link to the Creative Commons license, and indicate if changes were made. The images or other third party material in this article are included in the article's Creative Commons license, unless indicated otherwise in a credit line to the material. If material is not included in the article's Creative Commons license and your intended use is not permitted by statutory regulation or exceeds the permitted use, you will need to obtain permission directly from the copyright holder. To view a copy of this license, visit http://creativecommons. org/licenses/by/4.0/

\section{References}

1. Croker AK, Goodale D, Chu J, Postenka C, Hedley BD, Hess DA, et al. High aldehyde dehydrogenase and expression of cancer stem cell markers selects for breast cancer cells with enhanced malignant and metastatic ability. J Cell Mol Med. 2009;13:2236-52.

2. Al-Hajj M, Wicha MS, Benito-Hernandez A, Morrison SJ, Clarke MF. Prospective identification of tumorigenic breast cancer cells. Proc Natl Acad Sci USA. 2003;100:3983-8.

3. Reya T, Morrison SJ, Clarke MF, Weissman IL. Stem cells, cancer, and cancer stem cells. Nature. 2001;414:105-11.

4. Clark DW, Palle K. Aldehyde dehydrogenases in cancer stem cells: potential as therapeutic targets. Ann Transl Med. 2016;4:518-25.

5. Ginestier C, Hur MH, Charafe-Jauffret E, Monville F, Dutcher J, Brown $\mathrm{M}$, et al. ALDH1 is a marker of normal and malignant human mammary stem cells and a predictor of poor clinical outcome. Cell Stem Cell. 2007;1:555-67.

6. Petersen OW, Polyak K. Stem cells in the human breast. Cold Spring Harb Perspect Biol. 2010;2:a003160.

7. Cochrane CR, Szczepny A, Watkins DN, Cain JE. Hedgehog signaling in the maintenance of cancer stem cells. Cancers. 2015;7:1554-85

8. de Sousa EMF, Vermeulen L. Wnt signaling in cancer stem cell biology. Cancers. 2016;8:60-77.

9. Desai A, Yan Y, Gerson SL. Concise reviews: cancer stem cell targeted therapies: toward clinical success. Stem cells Transl Med. 2019;8:75-81.

10. Kahn M. Wnt signaling in stem cells and cancer stem cells: a tale two coactivators. Prog Mol Biol Transl Sci. 2018;153:209-44.

11. Kaltschmidt C, Banz-Jansen C, Benhidjeb T, Beshay M, Forster $\mathrm{C}$, Greiner J, et al. A role for NF-kappaB in organ specific cancer and cancer stem cells. Cancers. 2019;11:655-76.

12. Kroon P, Berry PA, Stower MJ, Rodrigues G, Mann VM, Simms $\mathrm{M}$, et al. JAK-STAT blockade inhibits tumor initiation and clonogenic recovery of prostate cancer stem-like cells. Cancer Res. 2013;73:5288-98.

13. Matsui WH. Cancer stem cell signaling pathways. Medicine. 2016;95:S8-19.
14. Chang WW, Lin RJ, Yu J, Chang WY, Fu CH, Lai AC, et al. The expression and significance of insulin-like growth factor-1 receptor and its pathway on breast cancer stem/progenitors. Breast Cancer Res. 2013;15:R39-54.

15. Chan YT, Lai AC, Lin RJ, Wang YH, Wang YT, Chang WW, et al. GPER-induced signaling is essential for the survival of breast cancer stem cells. Int $\mathrm{J}$ cancer. 2020;146:1674-85.

16. Zhang C, Kho YS, Wang Z, Chiang YT, Ng GK, Shaw PC, et al. Transmembrane and coiled-coil domain family 1 is a novel protein of the endoplasmic reticulum. PLoS ONE. 2014;9:e85206.

17. Hoyer MJ, Chitwood PJ, Ebmeier CC, Striepen JF, Qi RZ, Old WM, et al. A novel class of ER membrane proteins regulates ERassociated endosome fission. Cell. 2018;175:254-54.

18. Hopkins PC, Sainz-Fuertes R, Lovestone S. The impact of a novel apolipoprotein $\mathrm{E}$ and amyloid-beta protein precursor-interacting protein on the production of amyloid-beta. J Alzheimer's Dis. 2011;26:239-53.

19. Hopkins PC. Neurodegeneration in a Drosophila model for the function of TMCC2, an amyloid protein precursor-interacting and apolipoprotein E-binding protein. PLoS ONE. 2013;8: e55810.

20. Sohn W-J, Kim J-Y, Kim D, Park J-A, Lee Y, Kwon H-J. Expression and characterization of transmembrane and coiled-coil domain family 3. BMB Rep. 2016;49:629-34.

21. Saadin K, White IM. Breast cancer stem cell enrichment and isolation by mammosphere culture and its potential diagnostic applications. Expert Rev Mol Diagnostics. 2013;13:49-60.

22. Lombardo Y, de Giorgio A, Coombes CR, Stebbing J, Castellano L. Mammosphere formation assay from human breast cancer tissues and cell lines. J Vis Exp. 2015:e52671.

23. Wang T, Narayanaswamy R, Ren H, Torchilin VP. Combination therapy targeting both cancer stem-like cells and bulk tumor cells for improved efficacy of breast cancer treatment. Cancer Biol Ther. 2016;17:698-707.

24. Yin H, Glass J. The phenotypic radiation resistance of CD44+/CD24 (-or low) breast cancer cells is mediated through the enhanced activation of ATM signaling. PLoS ONE. 2011;6:e24080.

25. Brabletz T, Jung A, Spaderna S, Hlubek F, Kirchner T. Opinion: migrating cancer stem cells - an integrated concept of malignant tumour progression. Nat Rev Cancer. 2005;5:744-9.

26. Ayob AZ, Ramasamy TS. Cancer stem cells as key drivers of tumour progression. J Biomed Sci. 2018;25:20-37.

27. Chappell WH, Steelman LS, Long JM, Kempf RC, Abrams SL, Franklin RA, et al. Ras/Raf/MEK/ERK and PI3K/PTEN/Akt/ mTOR inhibitors: rationale and importance to inhibiting these pathways in human health. Oncotarget. 2011;2:135-64.

28. Iliopoulos D, Polytarchou C, Hatziapostolou M, Kottakis F, Maroulakou IG, Struhl K, et al. MicroRNAs differentially regulated by Akt isoforms control EMT and stem cell renewal in cancer cells. Sci Signal. 2009;2:ra62.

29. Irie HY, Pearline RV, Grueneberg D, Hsia M, Ravichandran P, Kothari N, et al. Distinct roles of Akt1 and Akt2 in regulating cell migration and epithelial-mesenchymal transition. J Cell Biol. 2005;171:1023-34.

30. Latifi A, Abubaker K, Castrechini N, Ward AC, Liongue C, Dobill $\mathrm{F}$, et al. Cisplatin treatment of primary and metastatic epithelial ovarian carcinomas generates residual cells with mesenchymal stem cell-like profile. J Cell Biochem. 2011;112:2850-64.

31. Li J, Zhou BP. Activation of beta-catenin and Akt pathways by Twist are critical for the maintenance of EMT associated cancer stem cell-like characters. BMC Cancer. 2011;11:49-59.

32. Wang YK, Zhu YL, Qiu FM, Zhang T, Chen ZG, Zheng S, et al. Activation of Akt and MAPK pathways enhances the tumorigenicity of CD133+ primary colon cancer cells. Carcinogenesis. 2010;31:1376-80. 
33. Kim LC, Cook RS, Chen J. mTORC1 and mTORC2 in cancer and the tumor microenvironment. Oncogene. 2016;36:2191-201.

34. Sarbassov DD, Guertin DA, Ali SM, Sabatini DM. Phosphorylation and regulation of Akt-PKB by the rictor-mTOR complex. Science. 2005;307:1098-101.

35. Prossomariti A, Piazzi G, Alquati C, Ricciardiello L. Are Wnt/ $\beta$-Catenin and PI3K/AKT/mTORC1 distinct pathways in colorectal cancer? Cell Mol Gastroenterol Hepatol. 2020;10:491-506.

36. Yeo HL, Fan TC, Lin RJ, Yu JC, Liao GS, Chen ES, et al. Sialylation of vasorin by ST3Gal1 facilitates TGF- $\beta 1$-mediated tumor angiogenesis and progression. Int $\mathrm{J}$ Cancer. 2019;144:1996-2007.

37. Kuo MW, Wang SH, Chang JC, Chang CH, Huang LJ, Lin HH, et al. A novel puf-A gene predicted from evolutionary analysis is involved in the development of eyes and primordial germ-cells. PLoS ONE. 2009;4:e4980.

38. Wisesa S, Yamamoto Y, Sakisaka T. TMCC3 localizes at the three-way junctions for the proper tubular network of the endoplasmic reticulum. Biochem J. 2019;476:3241-60.

39. Blume-Jensen P, Hunter T. Oncogenic kinase signalling. Nature. 2001;411:355-65.

40. Bose S, Chandran S, Mirocha JM, Bose N. The Akt pathway in human breast cancer: a tissue-array-based analysis. Mod Pathol. 2006;19:238-45.

41. Jain MV, Jangamreddy JR, Grabarek J, Schweizer F, Klonisch T, Cieslar-Pobuda A, et al. Nuclear localized Akt enhances breast cancer stem-like cells through counter-regulation of p21(Waf1/ Cip1) and p27(kip1). Cell Cycle. 2015;14:2109-20.

42. Bozorgi A, Khazaei M, Khazaei MR. New findings on breast cancer stem cells: a review. J Breast Cancer. 2015;18:303-12.

43. Yap TA, Bjerke L, Clarke PA, Workman P. Drugging PI3K in cancer: refining targets and therapeutic strategies. Curr Opin Pharmacol. 2015;23:98-107.
44. Liao Y, Hung MC. Physiological regulation of Akt activity and stability. Am J Transl Res. 2010;2:19-42.

45. Wang W, Shen T, Dong B, Creighton CJ, Meng Y, Zhou W, et al. MAPK4 overexpression promotes tumor progression via noncanonical activation of AKT/mTOR signaling. J Clin Investig. 2019;129:1015-29.

46. Swiatkowski P, Nikolaeva I, Kumar G, Zucco A, Akum BF, Patel $\mathrm{MV}$, et al. Role of Akt-independent mTORC1 and GSK3 $\beta$ signaling in sublethal NMDA-induced injury and the recovery of neuronal electrophysiology and survival. Sci Rep. 2017;7:1539-54.

47. Mahajan K, Mahajan NP. PI3K-independent AKT activation in cancers: a treasure trove for novel therapeutics. J Cell Physiol. 2012;227:3178-84.

48. Brugge J, Hung M-C, Mills GB. A new mutational aktivation in the PI3K pathway. Cancer Cell. 2007;12:104-7.

49. Yudushkin I. Getting the Akt together: guiding intracellular Akt activity by PI3K. Biomolecules. 2019;9:67-80.

50. Shorning BY, Dass MS, Smalley MJ, Pearson HB. The PI3KAKT-mTOR pathway and prostate cancer: at the crossroads of AR, MAPK, and WNT signaling. Int $\mathrm{J}$ Mol Sci. 2020;21:4507-53.

51. Kume H, Muraoka S, Kuga T, Adachi J, Narumi R, Watanabe S, et al. Discovery of colorectal cancer biomarker candidates by membrane proteomic analysis and subsequent verification using selected reaction monitoring (SRM) and tissue microarray (TMA) analysis. Mol Cell Proteom. 2014;13:1471-84.

52. Johnston HE, Carter MJ, Larrayoz M, Clarke J, Garbis SD, Oscier $\mathrm{D}$, et al. Proteomics profiling of CLL versus healthy B-cells identifies putative therapeutic targets and a subtype-independent signature of spliceosome dysregulation. Mol Cell Proteom. 2018;17:776-91.

53. Vieira AF, Schmitt F. An update on breast cancer multigene prognostic tests-emergent clinical biomarkers. Front Med. 2018;5:248-59. 